\title{
Healthy people with delusional ideation change their mind with conviction
}

\author{
Mitchell Rodier ${ }^{\mathrm{a}, \mathrm{c}, 1}$, Marie Prévost ${ }^{\mathrm{b}, \mathrm{c}, 1}$, Louis Renoult ${ }^{\mathrm{b}, \mathrm{c}}$, Claire Lionnet $^{\mathrm{c}}$, Yvonne Kwann ${ }^{\mathrm{c}}$, \\ Emmanuelle Dionne-Dostie ${ }^{\mathrm{c}}$, Isabelle Chapleau ${ }^{\mathrm{c}}$, J. Bruno Debruille ${ }^{\mathrm{a}, \mathrm{b}, \mathrm{c}, *}$ \\ a Department of Psychiatry, McGill University, Montreal, Qc, Canada \\ b Department of Neurology and Neurosurgery, McGill University, Montreal, QC, Canada \\ c Douglas Mental Health University Institute, Montreal, QC, Canada
}

\section{A R T I C L E I N F O}

\section{Article history:}

Received 31 August 2010

Received in revised form 2 June 2011

Accepted 22 June 2011

Available online $\mathrm{xxxx}$

\section{Keywords:}

Jump to conclusions

Reasoning

Delusions

Depression

Conviction

Schizotypy

\begin{abstract}
A B S T R A C T
Emotional distress and reasoning biases are two factors known to contribute to delusions. As a step towards elucidating mechanisms underlying delusions, the main aim of this study was to evaluate a possible "jumping to new conclusions" reasoning bias in healthy people with delusional ideation and its association with emotions. We surveyed 80 healthy participants, measuring levels of depression, anxiety, cognitive error and delusional ideation. Participants completed two versions of the beads task to evaluate their reasoning style. Results showed that people with delusional ideation reached a conclusion after less information, as expected. Interestingly, they also tended to change their conclusions more often than people without delusional ideation and did so with greater conviction. Depression and cognitive errors were strong predictors of delusional ideation but not of reasoning style. We conclude that delusional ideation in non-psychotic individuals is independently predicted by depressive symptoms and by a high conviction in new conclusions.
\end{abstract}

(c) 2011 Elsevier Ltd. All rights reserved.

\section{Introduction}

Delusional thinking has been the focus of many investigations and theoretical formulations in recent years (for reviews, see Garety and Hemsley, 1994; Bentall and Kinderman, 1998; Garety and Freeman, 1999; Bentall et al., 2001; Fine et al., 2007; Freeman, 2007). Compelling evidence suggests that people with delusional thinking have both a distinct reasoning style and a more affective symptomatology than people without delusions.

The distinct reasoning style seen in delusional patients is characterised by a tendency to 'jump to conclusions' (JTC). That is, they tend to draw conclusions based on less evidence than nondelusional participants (e.g., Huq et al., 1988; Garety et al., 1991; Garety et al., 2005; van Dael et al., 2006; Langdon et al., 2010; for a review see Fine et al., 2007). Even though the conclusions reached in the typical jump to conclusions paradigm are not of delusional content, such a mechanism of hasty decision-making can help explain why someone could consider a false belief in the first place (Ziegler et al., 2008). The same bias is observed in healthy people with delusional ideation (Colbert and Peters, 2002; McKay et al., 2006; Freeman et al., 2008; White and Mansell, 2009). Although delusions are typically observed as symptoms of psychiatric illnesses, similar beliefs

\footnotetext{
* Corresponding author at: Human Neurocognitive Science Laboratory, F. B. C. Pavilion, Douglas Hospital Research Centre, 6875 Blvd. LaSalle, Montréal, QC, H4H 1R3, Canada. Tel.: +1 514761 6131\#3405; fax: +1 5148884099.

E-mail address: bruno.debruille@mcgill.ca (J.B. Debruille).

${ }^{1}$ Both authors contributed equally to the manuscript.
}

(hereafter named delusional ideation) also manifest in the nonclinical population (Peters et al., 1999; Verdoux and van Os, 2002). This JTC bias is more likely to be involved in delusion formation than delusion maintenance, since it persists once delusions have remitted (Peters and Garety, 2006), albeit at a reduced level, and since most patients go on to develop delusions again in the future.

In an attempt to better define the JTC bias, Garety et al. (1991) investigated how deluded patients would react to disconfirmatory evidence. They found that patients showed a tendency to jump to new conclusions, as deluded schizophrenia patients strongly reacted to disconfirmatory evidence compared to controls. In a similar paradigm, Moritz and Woodward (2005) reported that schizophrenia patients were more swayed by evidence that contradicts their first conclusion. Indeed, delusional schizophrenia patients were more likely than nondelusional psychiatric controls and non-patients to reduce their level of certainty when presented with disconfirmatory evidence. Other studies did not find differences between the way delusional and control groups reacted to disconfirmatory evidence (Dudley et al., 1997; Fear and Healy, 1997; Young and Bentall, 1997; Peters and Garety, 2006). One study in healthy participant found that people with high scores of delusional ideation were also more reactive to a piece of disconfirmatory evidence than people with low scores (Colbert and Peters, 2002). However, most of these studies explored people's confidence levels for their initial choice in face of disconfirmatory evidence. They did not explore jumping to new conclusions per se. The jury is still out on whether delusional thinking is associated with an inclination to 'jump to new conclusions'. This possible bias has never been tested, to our knowledge, in healthy participants. One aim 
of the present study is thus to test the hypothesis that healthy people with delusional ideation would change their mind once presented with evidence that contradicts their initial conclusion and would do so in a hastier manner than people without delusional ideation. If such a bias were observed, this would support the idea that delusions could arise from an ability to jump from one (accurate) belief to another (inaccurate) with very little evidence.

More recent conceptualizations of the formation and maintenance of delusions consider the role of emotion. Several studies have demonstrated that low mood and low self-esteem, as well as negative schemas about the self and others, may be associated with the presence of psychotic symptoms (e.g. Close and Garety, 1998). Krabbendam et al. (2005) found that patients who were hallucinating at baseline were more likely to have psychotic symptoms 3 years later if they also exhibited depressed mood at baseline. Barrowclough et al. (2003) found that schizophrenia patients with a negative selfevaluation scored higher on the positive symptoms subscale measuring hallucinations and delusions. Smith et al. (2006) examined patients who suffered relapses in psychosis and showed that those with worse depression, self-esteem, and negative beliefs, had persecutory delusions of greater severity. In healthy participants, two longitudinal studies reported that psychosis-prone individuals had a higher rate of Major Depressive Disorder than controls (Chapman et al., 1994; Kwapil et al., 1997) and were at a higher risk to develop an incident depression (Verdoux et al., 1999). Low selfesteem was also associated to delusional ideation in the general population (Warman et al., 2010). As in patients, depression and anxiety scores seemed to be related to psychotic experiences (Lewandowski et al., 2006) and especially to subclinical paranoia in non-clinical populations (Combs and Penn, 2004).

Taken together, both reasoning biases and emotions seem to play a role in delusions. Bentall et al. (2009) observed in patients with paranoid delusions that emotions, and especially depression and negative thinking, were more strongly associated with delusions than reasoning bias. Studying deluded patients, Garety et al. (2005) reported a significant association between JTC and delusions, and between anxiety and delusions. However, the JTC was not associated to anxiety or depression, suggesting an independent role of reasoning processes and emotions in delusions. This view has been somewhat challenged by another study where patients with persecutory delusions display an increase of anxiety, paranoia, and of the tendency to jump to conclusion after exposure to urban environment (Ellett et al., 2008). However, no correlations were run in this study to evaluate whether the increased JTC was associated with the increased paranoia or/and the increased anxiety. However, it appears likely that the increased JTC was observed because of the increased paranoia. In healthy people, some found that inducing anxiety significantly increases the JTC bias and the levels of state paranoia in high scorers on the CAPE, a psychotic experiences questionnaire (Lincoln et al., 2010a, 2010b) whilst others did not find effect of induced anxiety on the JTC bias (Keefe and Warman, 2011). Given the lack of strong rationale behind an association between JTC and depression or anxiety, we hypothesise that delusions are independently related to JTC and emotions.

Another cognitive bias has been found in psychiatric patients, especially depressive patients. It consists of a tendency to form negative inferences and is measured by the cognitive errors questionnaire with items such as: You noticed recently that a lot of your friends are taking up golf and tennis. You would like to learn, but remember the difficulty you had that time you tried to ski. You think to yourself, "I couldn't learn skiing, so I doubt if I can learn to play tennis." This bias is associated with depression (Deal and Williams, 1988; Haaga et al., 1991). Surprisingly, no previous studies have assessed whether this tendency is more frequent in people with delusions or with delusional ideation. Moreover, the relation between this tendency and other cognitive bias such as the JTC has not been assessed. The present study assesses whether this type of cognitive errors could mediate a link between emotions such as depression, and delusional ideation. Freeman (2007) has proposed a model where emotions participate in delusion formation concurrently with reasoning biases. However, it remains unclear whether depression or anxiety is the best predictor of delusional ideation. Anxiety has been clearly linked to paranoia (Freeman et al., 2008; Lincoln et al., 2010a, 2010b), but depressive symptoms as well (Chapman et al., 1994; Kwapil et al., 1997; Warman et al., 2010). Therapy to improve anxiety differs greatly from that to improve depression. It is thus necessary to try to elucidate which of these emotional factors would be best to target concurrently with delusional symptoms. Based on Freeman's model, we examined non-clinical participants, which allows for the assessment of reasoning and emotional factors under conditions free from the effects of long-term illness and medication on mood and cognition. The aim of the present study was thus to test the following hypotheses in non-clinical people: 1 /delusional ideation is correlated with a data gathering bias and a tendency to change conclusion; 2/delusional ideation is correlated with anxiety and depression levels; $3 /$ the link between delusional ideation and reasoning bias is independent from that between delusional ideation and emotions and 4/cognitive errors might mediate a link between depression levels, JTC and delusional ideation.

\section{Methods}

\subsection{Participants}

Eighty (45 females) non-psychiatric participants, aged between 18 and 50 years $($ mean $=29.4$, S.D. $=9.7)$, were recruited using advertisements in two Montreal newspapers (one French and one English), as other studies did (Colbert and Peters, 2002; Freeman et al., 2008). Participants were screened by phone and asked if they had already been diagnosed with any psychiatric disorder or psychological problems. The same questions were asked about their close relatives. They were also asked if they were taking any medication at the time of the study or in the past. Those who reported past or current diagnosed psychiatric disorders, close relative(s) with schizophrenia or bipolar disorder, neurological disease, psychotropic drug use, psychiatric medication use and substance use disorders were excluded. All participants had a minimum of college level education. The procedure to recruit the participants included a quick questionnaire made of 16 items from the schizotypal personality questionnaire (SPQ Raine, 1991; French version: Dumas et al., 1999) that was used to pre-assess delusional ideation over the phone. These 16 items constitute two subscales of the SPQ described by Raine et al. (1994) as 'ideas of reference' and 'odd beliefs and magical thinking'. In the first phase of the recruitment, only 35 participants who scored 5 or more out of 16 (the maximum score), were asked to participate in the study, in order to have enough participants with high delusional ideation scores. This was based on a pilot study that showed that recruiting without screening for delusional ideation results in obtaining a vast majority of participants scoring very low for delusional ideation and having not enough variance for correlation analyses. Even with our precaution, our total sample mean score at the Peters et al. Delusion Inventory (PDI-21) was 5.4 (S.D. = 3.6), which is slightly lower than Peters et al.'s mean of 6.7 (S.D. =4.4) (2004). Forty-five no- or low-delusional ideation participants were recruited in a second phase amongst people with delusional ideation scores smaller than five who had been screened during the first phase. The pilot study had also showed that participants' answers for the delusional ideation questions of the SPQ sometimes changed between phone screening and on site testing. We thus chose to use two different questionnaires: the delusional ideation subscale of the SPQ for phone screening, and the PDI-21 for data collection, the later being more specific of delusional ideation.

Each participant gave written informed consent in accordance with the Douglas Institute Research Ethics Board's criteria. Participants were financially compensated for their time.

\subsection{Questionnaires}

All questionnaires were presented in the participants' mother tongue or their preferred written language if considered fully bilingual (English or French only).

Delusional ideation was assessed using the Peters et al. Delusions Inventory (PDI21, Peters et al., 2004) which includes 21 questions asking whether or not the participant has a particular idea. Delusional ideation was measured using the total number of Yes responses on the PDI-21 (range is 0 to 21). The French version was that used previously by Verdoux et al. $(1998,1999)$ and van Os et al. (1999).

To estimate participants' emotional states, we administered the Beck Depression Inventory (Beck et al., 1961, French version: Bourque and Beaudette, 1982) and the 'State' part of the State-Trait Anxiety Inventory form Y (STAI, Spielberger et al., 1983; French version: Bruchon-Schweitzer and Paulhan, 1993), which we will refer to herein 
as the SAI. Total scores for the SAI (range 0 to 60 ) were used as a measure of anxiety level and total scores for the Beck Depression Inventory (range 0 to 63) were used as a measure of depression level.

The Cognitive Error Questionnaire (CEQ Lefebvre, 1981 for both language versions) was administered to measure the tendency for common cognitive errors or distorted beliefs. This questionnaire was added to evaluate the relationship between this tendency and the other reasoning anomaly tested that is the jumping to conclusion bias. Concurrently, it was of interest to explore a possible association of cognitive errors that are acknowledgedly linked to depression (Lefebvre, 1981; Pössel, 2009) with delusional ideation. In this questionnaire, participants are presented a series of short vignettes followed by a dysphoric cognition. For example, item 1 is as follows: 'Your boss just told you that because of a general slowdown in the industry, he has to lay off all of the people who do your job including you. You think to yourself, "I must be doing a lousy job or else he wouldn't have laid me off." Participants are asked to what degree they would have thought the same thing (on a five-point scale). Scores generated were the sum of all five-point scores (range 0 to 96 ).

Intelligence was estimated using the short form of the verbal subtest of the Wechsler Adult Intelligence Scale-III (WAIS-III, Wechsler, 1997; French version: Wechsler, 2000). The WAIS-S subscale examines abstract thinking by a test of Similarities between items. The total score of this subscale ranges from 0 to 38 . The WAIS-K subscale looks at level of Knowledge base in a variety of domains. The total score of this subscale ranges from 0 to 28 . Measures of intelligence were collected as Lincoln et al. (2010a, 2010b) reported that the relationship between a hasty decision making and delusions in patients was cancelled if IQ was controlled for. In addition, in healthy people, Freeman et al. (2008) reported a trend to observe lower IQ in those who jump to conclusions relative to those who did not and Colbert and Peters (2002 reported a negative correlation between IQ and change to disconfirmatory evidence. It was thus necessary to evaluate whether intelligence could account for some of the variability in the beads task performance.

\subsection{Beads task}

We used a beads task design based on that of Garety et al. (1991) to measure participants' tendency to 'jump-to-conclusions'. The task was run using a Microsoft PowerPoint slideshow. Participants were presented with two jars: jar A contains 85\% of green beads and $15 \%$ of yellow beads, whereas jar B has the opposite proportions. Beads were drawn from one of the two jars and the participant had to guess the jar from which the beads were drawn. All participants were provided a specific fixed sequence (Fig. 1), as described in Colbert and Peters (2002). The sequence was stopped as soon as the participant reached a conclusion and was as certain as possible about the jar of origin. The task was conducted first as a practice run (with different colours) and then again for data collection (Part 1). Participants were given basic feedback (examiner repeated the instructions to the participants, stating that all beads come from a single jar) during the practice sequence to optimise their understanding of the task.

Part 2 of the beads task tested participants' tendencies to maintain or change their conclusions in the face of disconfirmatory evidence. Participants were presented with a new sequence of 38 beads and were told that all beads were coming from a single jar, as in Part 1. However, the first half of the sequence had a majority of green beads, suggesting jar A, whereas the second half had a majority of yellow beads, suggesting jar B (Fig. 1; see Garety et al., 1991; Langdon et al., 2010). At the end of the sequence, exactly $50 \%$ of the beads drawn were yellow and $50 \%$ were green, making the choice of jar A or B equally likely. Participants were asked to guess after each bead from which jar the beads were being drawn and how certain they were of their guess. As such,
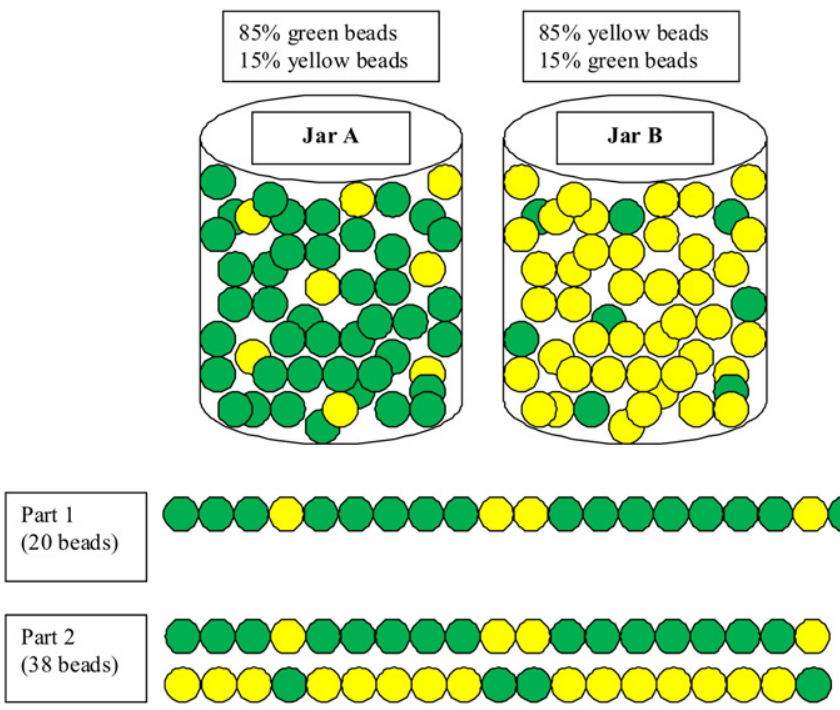

Fig. 1. Summary of the sequences used for the beads task. certainty levels measured in the present study reflect the certainty with which participants were choosing the jar of origin for each bead presented. It is important to note that although this beads sequence would appear extremely unlikely, all participants carried out the task without questioning the likelihood of the sequence.

For part 1 of the beads task, we measured the number of beads presented before a decision was made; this was termed the draws to decision (DTD) and this variable ranged from 1 (for participants who choose a jar at the first bead) to 21 (for participants who choose a jar after seeing all the beads). For part 2 of the beads task, certainty percentages were collected for each bead, as in Fear and Healy (1997). This yielded four measures: Max1, Max2, Jar Change, and Beads to Change (BTC). The Max1 and Max2 measures were based on the hypothesis that certainty values on the beads task are related to a person's degree of conviction in beliefs. We examined these potential correlates of conviction during the accumulation of supporting (Max1) and disconfirmatory (Max2) evidence. Max1 corresponded to the maximum certainty (\%) attained in the first half of the sequence (a measure that was sometimes found to be related to delusional-like ideation, e.g., Warman, 2008). Max2 corresponded to the maximum certainty (\%) attained in the second half of the sequence for participants who switched to the alternate jar. We also examined two measures of belief reversal as a way of testing the degree of belief rigidity. The first was the occurrence of a change of belief about which jar was being used ('Jar Change'). The second was the BTC which corresponded to the number of beads needed to change jars in the second half of the sequence and was our variable to assess the tendency to jump to new conclusions. In the present study, the data-gathering bias is quantified by the negative correlation between PDI-21 scores and DTD. A tendency to jump to new conclusions is defined by a significant negative correlation between PDI scores and BTC.

\subsection{Analyses and statistics}

The hypothesis that delusional ideation was correlated with distinct reasoning styles and emotional states was tested by examining correlations between PDI-21 scores and both performance measures on the beads tasks and measures of emotional states. Non-parametric Spearman correlations were run because Beck, DTD, BTC and Max1 scores were not normally distributed, as tested with the Kolmogorov-Smirnov test (Beck: $D=0.208, p<0.001$; DTD: $D=0.223, p<0.001$; BTC: $D=0.183, p=0.008$; Max1: $D=0.0237, p<0.001)$. All results are two-tailed.

Once significant correlations were uncovered, each significant factor was entered in a stepwise manner into a linear regression model. Two linear regressions were run to test which factors predicted PDI-21 scores: the first one with all participants and the second one with the 32 participants who switched jar in Part 2. Kolmogorov-Smirnov tests showed that the distributions of the residuals in both regressions did not differ significantly from a normal distribution (1st regression: $D=0.076, p=0.200$; 2nd regression: $D=0.140, p=0.110$ ), allowing the use of this model with our data. It is worth noting that the choice to add only significant correlating variables in the linear regression does not exclude the possibility that other variables could explain delusional ideation. Independent samples Mann Whitney $U$ test was used to analyse the differences found between the means of a measure when there was a clear need to divide the population into two groups: for those who switched jars vs. those who did not in the second half of the Part 2 sequence and for those with a JTC (decision reached after one or two beads) vs. the other participants.

\section{Results}

\subsection{Means observed in the population sample}

The mean PDI-21 score was 5.4 (S.D. = 3.6). The mean score for depression was 3.5 (S.D. $=4.3$ ), for anxiety 24.3 (S.D. $=13.3$ ) and for

Table 1

Spearman's correlations, indicated as rho (p).

\begin{tabular}{lclll}
\hline$N=80$ & PDI-21 & SAI & CEQ & Beck \\
\hline DTD & $-\mathbf{0 . 2 2}(\mathbf{0 . 0 4 5})$ & $0.16(0.16)$ & $-0.063(0.58)$ & $-0.082(0.47)$ \\
Max1 & $0.12(0.29)$ & $-0.005(0.96)$ & $0.14(0.20)$ & $-0.028(0.81)$ \\
Max2 $^{\mathrm{a}}$ & $\mathbf{0 . 3 9}(\mathbf{0 . 0 2 9})$ & $-0.19(0.29)$ & $-0.23(0.21)$ & $0.063(0.73)$ \\
BTC $^{\mathrm{a}}$ & $-0.16(0.36)$ & $-0.25(0.17)$ & $-0.14(0.45)$ & $-\mathbf{0 . 3 5}(\mathbf{0 . 0 4 9})$ \\
Beck & $\mathbf{0 . 4 6}(<\mathbf{0 . 0 0 1})$ & $\mathbf{0 . 2 6}(\mathbf{0 . 0 1 8})$ & $\mathbf{0 . 3 6}(\mathbf{0 . 0 0 1})$ & 1 \\
CEQ & $\mathbf{0 . 2 9}(\mathbf{0 . 0 0 8})$ & $\mathbf{0 . 3 5}(\mathbf{0 . 0 0 2})$ & 1 & \\
SAI & $0.063(0.58)$ & 1 & &
\end{tabular}

Boldface indicates $p<.05$

$\mathrm{CEQ}=$ Cognitive Error Questionnaire; Beck = Beck Depression Score; SAI = State part of the State/Trait Anxiety Inventory; PDI-21 = Peters et al. Delusions Inventory; DTD = Draws to Decision; Max1 = Maximum certainty (\%) attained in first half of the sequence; Max2 = Maximum certainty (\%) attained in second half of the sequence; BTC = number of beads needed to change jar in the second half of the sequence.

The alpha level after Bonferroni corrections would have been set at 0.006 .

a Max2 and BTC correlations apply only to subjects who switched jars, $N=32$. 
cognitive errors 14 (S.D. $=9.7)$. The mean for the WAIS-similarities was 23.5 out of 38 (S.D. $=3.9$ ) and was 19.1 out of 28 (S.D. $=4.3$ ) for the WAIS-knowledge.

\subsection{Tendency to jump to conclusions}

The mean draws to decisions (DTD) was 9.2 (S.D. =7.3) and its median was 7 , which is rather high, mainly due to 10 participants deciding only after having seen the total sequence of beads. We found a negative correlation between the number of draws to decision (DTD) and PDI-21 (Table 1). DTD was also correlated with WAIS-S $(r=0.33, p=0.003)$. To check whether intelligence accounted for the relationship between DTD and PDI-21, we ran a 2-step linear regression to predict PDI-21 using DTD as the first step and WAIS-S as the second step. DTD remained a significant predictor of PDI-21 measures even after intelligence was controlled for $(p<0.05)$. There were no correlations between DTD and Beck, SAI, or CEQ. When we looked at the maximum percentage of certainty (Max1) reached in the first half of the part- 2 sequence ( mean $=87.2$, S.D. $=17.8$ ), no correlation was found between these certainty values and delusional ideation, showing that people with delusional ideation were not more certain of the jar they choose than people without delusional ideation.

Those who chose a jar after one or two beads $(N=11)$ scored significant higher on the PDI-21 $(U=158, p=0.002)$ and the Beck $(U=202, p=0.014)$, and lower on the WAIS-S $(U=225, p=0.03)$ than those who chose a jar after three or more beads. There were no other significant differences between these two groups.

\subsection{Tendency to jump to new conclusions and change of beliefs}

Participants who changed jars in the second half of the sequence of Part 2 tended to score higher on the PDI-21 (Mann Whitney $U=966$, $p=0.051)$. In other words, people with delusional ideation changed their conclusion more so than people without delusional ideation. Table 1 shows that the strongest correlation regarding the change of belief hypothesis was between PDI-21 and the maximum percentage of certainty reached after switching jars (Max2, mean $=72.4$, S.D. $=19.3$ ). The more participants believed their delusional ideation, the more certain they became that the alternate jar was being used in the second half of the part- 2 sequence. There were no significant correlations between intelligence estimates and Max2. It should be noted that only participants who changed their mind about which jar was used were included in these analyses $(N=32)$. Interestingly, the BTC (the number of beads needed to change jar in the Part 2, mean $=28.6$, S.D. $=5.8$ ) was positively correlated to the DTD (number of beads needed to reach a decision in the Part 1 ) (Spearman's $r=0.563, p<0.001$ ), showing that the tendency to jump to conclusions is associated with a tendency to jump to new conclusions. However, BTC did not correlate with the PDI-21.

\section{Table 2}

Linear regression models predicting PDI-21 scores.

\begin{tabular}{|c|c|c|c|}
\hline & Model & $r(p)$ & $\beta(p)$ \\
\hline \multicolumn{4}{|c|}{ Including all participants, $n=80$} \\
\hline \multirow[t]{2}{*}{ PDI-21 } & Beck & $0.43(<0.001)$ & $0.43(<0.001)$ \\
\hline & Beck, DTD & $0.49(<0.001)$ & $0.44(<0.001),-0.24(0.021)$ \\
\hline \multicolumn{4}{|c|}{ Excluding those who stayed with original jar, $n=32$} \\
\hline \multirow[t]{2}{*}{ PDI-21 } & Max2 & $0.42(0.017)$ & $0.42(0.017)$ \\
\hline & Max2, Beck & $0.56(0.004)$ & 0.397 (0.015), $0.373(0.022)$ \\
\hline
\end{tabular}

PDI-21 = Peters et al. Delusions Inventory; Max2 = Maximum certainty (\%) attained in second half of the sequence; Beck = Beck Depression Score; DTD = Draws to Decision; $r=$ correlation coefficient between the independent variable and delusional ideation scales; $\beta=$ coefficient correlation between each independent variable and delusional ideation scales, taking into account the other variables.
3.4. Emotion as it relates to delusional ideation and beads task performance

Our measures of 'emotion' were the scores for depression, cognitive error, and anxiety. Table 1 displays several correlations between Beck scores and PDI-21. As expected, Beck scores were positively correlated with the degree of cognitive error (CEQ) and anxiety levels (SAI). Table 1 shows positive correlations between cognitive error and delusional ideation. CEQ levels were correlated with SAI. Most interestingly, neither depression nor anxiety correlated with DTD or certainty levels. Depression scores were negatively correlated with BTC, indicating that amongst those who changed their choice of jar, the higher their score of depression, the smaller the number of beads required to change jar.

\subsection{Models predicting delusional thinking}

To investigate which variables were the best predictors of delusional ideation, we ran two regression analyses (Table 2). The dependent variables were set as PDI-21. Independent variables were entered in a stepwise manner and included all variables that significantly correlated with delusional ideation, which are Beck, CEQ, DTD and Max2 scores. Collinearity was assessed and tolerance coefficients ranged between 0.8 and 1 , suggesting that the influence of each independent variable on PDI-21 scores can be assessed with the linear regression.

In a first analysis, all participants $(N=80)$ were included and thus Max2 was excluded as it only applied to the 32 participants who changed their mind about the jar used. PDI- 21 was best predicted by depression and DTD.

In the second analysis which included only the 32 participants who changed jars, the two significant predictors of PDI-21 were Max2 and depression (Table 2). Taken together, these two factors accounted for $31.4 \%$ of the variance in delusional ideation scores. Cognitive errors did not emerge as a factor contributing to delusional ideation in the model.

\section{Discussion}

We investigated the relationship between delusional thinking, reasoning styles, and emotional states in a non-psychiatric population. Delusional thinking was associated with a hasty decision making, a tendency to change conclusions and, in those who changed their conclusion, to do so with conviction. The only emotional factor that correlated with delusional ideation was depression. We then examined the relative contributions of these factors in predicting delusional thinking. We found that hasty decision making and depression symptoms both contributed independently to the likelihood of having delusional ideation.

We replicated the finding of Colbert and Peters (2002) that nonclinical participants who are more delusional gather less evidence before drawing a conclusion than those who have few or no delusional ideas. Participants who scored higher on the measures quantifying delusional ideation requested fewer beads before deciding which jar the beads were coming from. The mean DTD (9.2) was higher than what has been observed in other studies (e.g., between 5.7 and 6.5 in Warman and Martin, 2006; between 2.3 and 4 in White and Mansell, 2009). This rather cautious number of beads requested before making a decision was partially due to the 10 participants choosing a jar only after seeing all the beads of the sequence. Excluding these 10 participants gives a mean DTD of 7.1, still a high number for this task. The other reason for this elevated mean DTD might have been the practice run that made participants more cautious by emphasising the importance of the task. However, others have reported mean DTDs as elevated as ours for the same task (e.g., 8.7 for low PDI-21 scorers in Colbert and Peters, 2002; 10.2 for non- 
deluded patients in Peters et al., 2008). The mean PDI-21 score of our sample (5.4) was lower than what has been reported by the creators of the PDI-21 (6.7 in Peters et al., 2004), thus possibly reducing the likelihood of very few DTD. In any case, after 2.2 beads drawn, the probability for one jar is $97 \%$ and thus a decision reached at this point would be acceptable in Bayesian terms. This means that the general population is usually overly cautious in this task (Garety and Freeman, 1999).

In the second half of the sequence of beads where it seemed that beads were coming from a different jar than suggested by the first half of the sequence, novel findings emerged. Participants who changed their belief about which jar was being used were more likely to have higher delusional ideation scores. People with delusional ideation were thus more prone to change their conclusion. In addition, the more delusional ideas they had, the more convinced they were of their new conclusions. However, they did not display a hasty decision making in their change of mind, suggesting that delusions may not be associated with a tendency to jump to new conclusions, but rather with a tendency to change conclusions. The concept of jumping to new conclusions has been considered with reservation (Fine et al., 2007) as it is often regarded as being at odds with an element of the usual definition of delusions: that they are "fixed false beliefs" (Sadock and Sadock, 2003). However, if people with delusions and people with delusional ideation jump to one conclusion, it had to be tested whether they jump again to another conclusion as quickly. It seems that they do not, but our results also show that they do not 'stick' to their first conclusion. The fixity of the belief might be underlain by other mechanisms and likely be specific to the delusional belief itself. Interestingly, in face of evidence contradicting their first conclusion, participants with high delusional ideation scores tended to change their conclusion. This result is in opposition with the idea that they have a 'bias against contradictory evidence' or BADE (Woodward et al., 2006a, 2007). In our study, it seems that they considered disconfirmatory evidence as having more weight than people with few or no delusional ideas.

The more-delusional participants who considered an alternate hypothesis did so with a greater sense of certainty (i.e., higher Max2). Together with the change of conclusion, the strong correlation we found between this measure and the PDI-21 scores is reminiscent of the results of Woodward et al. (2006b). These authors showed that schizophrenia patients exhibited a hindsight bias, that is, a tendency to disregard past errors and act as though they "knew it all along" (the 'KIA effect'). The patients in that study were inclined to place more importance on the evidence that was currently presented whilst disregarding previous accumulations of evidence, reminiscent of the model of schizophrenia proposed by Hemsley (1987). This is similar to our findings in participants with delusional ideation where they were more likely to disregard their first hypothesis and become highly confident of an alternate hypothesis. It supports the idea that delusional thinking is related to a tendency to attach too much importance to current/recent evidence whilst relatively ignoring past events. Kapur (2003) proposed that delusions are associated with an aberrant salience of events or experiences. In the beads task, people with delusional ideation might reach a conclusion quickly and be highly confident about their new conclusion because of the exaggerated salience each bead carries for them.

Our results regarding the link between emotional measures and delusional thinking are in line with previous studies. Depression scores correlated with PDI-21, a result that echoes findings in psychotic patients, reviewed by Freeman and Garety (2003), as well as findings in healthy people (Combs and Penn, 2004). In this current cross-sectional study, 'cause and effect' of this association cannot be evaluated, however, it is interesting to note that this correlation exists in a healthy population sample, suggesting that non-clinical emotional disturbances might play a role in the formation or maintenance of delusional thoughts.
As expected, Beck depression scores correlated with levels of cognitive error (CEQ). This is in accordance with the claim of Beck et al. (1979) that depression is associated with negativistic errors in thinking. This claim has been supported by empirical studies that examined the association between cognitive errors and depressed mood (e.g. Deal and Williams, 1988; Haaga et al., 1991). CEQ scores also positively correlated with the PDI-21 scores. Cognitive errors could be considered an intermediate measure between reasoning bias (distorted appraisals of the likelihood of negative events) and emotional bias (the affective component of the cognitive error). Nevertheless, cognitive error was not predictive of delusional ideation after accounting for the Beck depression scores (Table 2). Accordingly, cognitive error probably does not mediate the link between depression and delusional ideation. Contrary to our hypothesis, CEQ did not correlate with any of the measures in the beads task, suggesting that the reasoning bias assessed in the CEQ is different to that assessed with the beads task. It is likely that the cognitive errors are strongly shaped by depression rather than by a reasoning abnormality.

The final measure of emotion that was examined was state anxiety level. As expected, SAI was highly correlated with depression scores and with CEQ. However, we found no relation between anxiety levels and performance on the beads task, supporting the study of So et al. (2008) which found no impact of anxiety manipulation on the JTC. Other investigators have found that in actively delusional patients, both the JTC bias and anxiety contributed independently to delusions (Garety et al., 2005; Bentall et al., 2009). This is in contrast to the present study where depression, rather than anxiety, was a significant variable. It is possible that anxiety plays a larger role in active clinical delusions than in non-clinical delusional ideation, making anxiety a possible marker of clinical delusions. Moreover, as proposed by Freeman (2007), anxiety might have a stronger role in delusion maintenance where the purpose of the delusional belief could be to lower the anxiety levels in patients. Interestingly, induced anxiety states are associated with enhanced JTC bias and with paranoid ideation in healthy people (Lincoln et al., 2010a, 2010b). In healthy participants, this relationship between delusional ideas and anxiety might be dependent of the environmental condition and be triggered in specific situations only.

Similarly to our results, Bentall et al. (2009) reported a strong association between paranoid delusions and measures of emotions, where emotions seemed independent of reasoning style. In the present study, the association between emotions and delusions appeared stronger than that between reasoning and delusions. Indeed, the regression analysis showed that depression was the variable that best predicts delusional ideation and explains the most variance. However, in both cases, these findings are in accordance with the relative null effect that emotional valence has on JTC (for a review, see Fine et al., 2007).

Not surprisingly, the reasoning element that was found to be most invoked in predicting delusional thoughts was the classic number of 'draws to decision', that is, the data gathering bias variable. However, when testing only people who change their conclusion, the best predictor of total delusional ideation scores and delusional ideation conviction was Max2, a variable reflecting the certainty with which one reaches new conclusions. As this variable has never been studied before, comparison with literature is difficult. Interestingly, Max2 explained the most variance for total scores of delusional ideation, cancelling the impact of depression in these cases.

A number of limitations have to be pointed out. First, the present study being cross-sectional, causes and consequences are only speculative. Second, a large number of correlations were run which enhanced the possibility of type I errors. A Bonferroni correction would have inversely enhanced the risk of hiding significant findings. Until these results are replicated in healthy people, the present conclusions should be taken with cautious. Finally, the present results 
were obtained in a non-clinical group having more or less delusionallike ideation. Thus, despite the fact that the continuum between psychosis and normality has been repetitively supported (Peters et al., 1999; van Os et al., 1999; Johns and van Os, 2001; Verdoux and van Os, 2002), the extrapolations to clinical groups have to be taken with great caution.

In conclusion, in addition to replicating the hasty decision making phenomenon in non-clinical individuals with delusional ideation, we showed that this population is more likely to change their conclusions and to develop an unusually high degree of conviction about alternate, new beliefs. Even though the jump to new conclusions style is not supported by our results, it is the first time that delusional ideation is associated with a tendency to change conclusions in healthy participants. Interestingly, this tendency should support the idea that people with delusional ideas would jump to a first conclusion and then change their mind, not 'sticking' to one conclusion or one belief. The maintenance of the delusional idea is thus likely to rest on other mechanisms, possibly on emotional ones. Delusional ideation was associated with levels of depression, but this association was not mediated by a distinct reasoning style. Emotional and reasoning factors emerged as independent factors predicting delusional thinking. With further elaborations of the distinct reasoning style of mildly delusional individuals and delusional patients, more refined approaches to cognitive behavioural therapy may be developed.

\section{Acknowledgements}

We would like to thank Dr Norbert Schmitz for his assistance with the statistical analyses conducted in this study. The study was run with a NARSAD grant allocated to J. Bruno Debruille who was supported by the 10084 FRSQ senior clinician researcher award. Marie Prévost was supported by a McGill returning student award.

\section{References}

Barrowclough, C., Tarrier, N., Humphreys, L., Ward, J., Gregg, L., Andrews, B., 2003. Selfesteem in schizophrenia: relationships between self-evaluation, family attitudes, and symptomatology. Journal of Abnormal Psychology 112, 92-99.

Beck, A.T., Ward, C., Mendelson, M., 1961. An inventory for measuring depression. Archives of General Psychiatry 4, 561-571.

Beck, A.T., Rush, A.J., Shaw, B., Emery, G., 1979. Cognitive Therapy for Depression. Guilford Press, New York, NY.

Bentall, R.P., Kinderman, P., 1998. Psychological processes and delusional beliefs: implications for the treatment of paranoid states. In: Wykes, T., Tarrier, N., Lewis, S. (Eds.), Outcome and Innovation in Psychological Treatment of Schizophrenia. Wiley Sons Ltd.

Bentall, R.P., Corcoran, R., Howard, R., Blackwood, N., Kinderman, P., 2001. Persecutory delusions: a review and theoretical integration. Clinical Psychology Review 21, 1143-1192.

Bentall, R.P., Rowse, G., Shryane, N., Kinderman, P., Howard, R., Blackwood, N., Moore, R., Corcoran, R., 2009. The cognitive and affective structure of paranoid delusions: a transdiagnostic investigation of patients with schizophrenia spectrum disorders and depression. Archives of General Psychiatry 66, 236-247.

Bourque, P., Beaudette, D., 1982. Étude psychométrique du questionnaire de dépression de Beck auprès d'un échantillon d'étudiants universitaires francophones. Revue Canadienne des Sciences du Comportement 14, 211-218.

Bruchon-Schweitzer, M.L., Paulhan, I., 1993. Manual of C. D. Spielberger's STAI-Y, French Adaptation. Editions du Centre de Psychologie Appliquée, Paris.

Chapman, L.J., Chapman, J.P., Kwapil, T.R., Eckblad, M., Zinser, M., 1994. Putatively psychosis-prone subjects 10 years later. Journal of Abnormal Psychology 103, 171-183.

Close, H., Garety, P.A., 1998. Cognitive assessment of voices: further developments in understanding the emotional impact of voices. The British Journal of Clinical Psychology 37, 173-188

Colbert, S.M., Peters, E.R., 2002. Need for closure and jumping-to-conclusions in delusion-prone individuals. The Journal of Nervous and Mental Disease 190, 27-31.

Combs, D.R., Penn, D.L., 2004. The role of subclinical paranoia on social perception and behavior. Schizophrenia Research 69, 93-104.

Deal, S.L., Williams, J.E., 1988. Cognitive distortions as mediators between life stress and depression in adolescents. Adolescence 23, 477-490.

Dudley, R.E., John, C.H., Young, A.W., Over, D.E., 1997. The effect of self-referent material on the reasoning of people with delusions. The British Journal of Clinical Psychology $36,575-584$.

Dumas, P., Rosenfeld, F., Saoud, M., Dalery, J., d'Amato, T., 1999. Translation and French adaptation of the Raine Schizotypal Personality Questionnaire. Encephale 25, 315-322.
Ellett, L., Freeman, D., Garety, P.A., 2008. The psychological effect of an urban environment on individuals with persecutory delusions: the Camberwell walk study. Schizophrenia Research 99, 77-84.

Fear, C.F., Healy, D., 1997. Probabilistic reasoning in obsessive-compulsive and delusional disorders. Psychological Medicine 27, 199-208.

Fine, C., Gardner, M., Craigie, J., Gold, I., 2007. Hopping, skipping or jumping to conclusions? Clarifying the role of the JTC bias in delusions. Cognitive Neuropsychiatry $12,46-77$.

Freeman, D., 2007. Suspicious minds: the psychology of persecutory delusions. Clinical Psychology Review 27, 425-457.

Freeman, D., Garety, P.A., 2003. Connecting neurosis and psychosis: the direct influence of emotion on delusions and hallucinations. Behaviour Research and Therapy 41, 923-947.

Freeman, D., Pugh, K., Garety, P., 2008. Jumping to conclusions and paranoid ideation in the general population. Schizophrenia Research 102, 254-260.

Garety, P.A., Freeman, D., 1999. Cognitive approaches to delusions: a critical review of theories and evidence. The British Journal of Clinical Psychology 38, 113-154.

Garety, P.A., Hemsley, D.R., 1994. Delusions: Investigations into the Psychology of Delusional Reasoning. Psychology Press, Hove.

Garety, P.A., Hemsley, D.R., Wessely, S., 1991. Reasoning in deluded schizophrenic and paranoid patients: biases in performance on a probabilistic inference task. The Journal of Nervous and Mental Disease 179, 194-201.

Garety, P.A., Freeman, D., Jolley, S., Dunn, G., Bebbington, P.E., Fowler, D.G., Kuipers, E., Dudley, R., 2005. Reasoning, emotions, and delusional conviction in psychosis. Journal of Abnormal Psychology 114, 373-384.

Haaga, D.A., Dyck, M.J., Ernst, D., 1991. Empirical status of cognitive theory of depression. Psychological Bulletin 110, 215-236.

Hemsley, D.R., 1987. An experimental psychological model for schizophrenia. In: Hafner, H., Gattaz, W.F., Janzarik, W. (Eds.), Search for the Causes of Schizophrenia. Springer, Heidelberg.

Huq, S.F., Garety, P.A., Hemsley, D.R., 1988. Probabilistic judgments in deluded and non-deluded subjects. The Quarterly Journal of Experimental Psychology 40, 801-812.

Johns, L.C., van Os, J., 2001. The continuity of psychotic experiences in the general population. Clinical Psychology Review 21, 1125-1141.

Kapur, S., 2003. Psychosis as a state of aberrant salience: a framework linking biology, phenomenology, and pharmacology in schizophrenia. The American Journal of Psychiatry 160 (1), 13-23.

Keefe, K.M., Warman, D.M., 2011. Reasoning, delusion proneness and stress: an experimental investigation. Clinical Psychology and Psychotherapy. 18, 138-147.

Krabbendam, L., Myin-Germeys, I., Hanssen, M., de Graaf, R., Vollebergh, W., Bak, M. van Os, J., 2005. Development of depressed mood predicts onset of psychotic disorder in individuals who report hallucinatory experiences. The British Journal of Clinical Psychology 44, 113-125.

Kwapil, T.R., Miller, M.B., Zinser, M.C., Chapman, J., Chapman, L.J., 1997. Magical ideation and social anhedonia as predictors of psychosis proneness: a partial replication. Journal of Abnormal Psychology 106, 491-495.

Langdon, R., Ward, P.B., Coltheart, M., 2010. Reasoning anomalies associated with delusions in schizophrenia. Schizophrenia Bulletin 36, 321-330.

Lefebvre, M.F., 1981. Cognitive distortion and cognitive errors in depressed psychiatric and low back pain patients. Journal of Consulting and Clinical Psychology 49 517-525.

Lewandowski, K.E., Barrantes-Vidal, N., Nelson-Gray, R.O., Clancy, C., Kepley, H.O. Kwapil, T.R., 2006. Anxiety and depression symptoms in psychometrically identified schizotypy. Schizophrenia Research 83, 225-235.

Lincoln, T.M., Lange, J., Burau, J., Exner, C., Moritz, S., 2010a. The effect of state anxiety on paranoid ideation and jumping to conclusions. An experimental investigation. Schizophrenia Bulletin 36, 1140-1148.

Lincoln, T.M., Ziegler, M., Mehl, S., Rief, W., 2010b. The jumping to conclusions bias in delusions: specificity and changeability. Journal of Abnormal Psychology 119 (1), 40-49.

McKay, R., Langdon, R., Coltheart, M., 2006. Need for closure, jumping to conclusions, and decisiveness in delusion-prone individuals. The Journal of Nervous and Mental Disease 194, 422-426.

Moritz, S., Woodward, T.S., 2005. Jumping to conclusions in delusional and nondelusional schizophrenic patients. The British Journal of Clinical Psychology 44, 193-207.

Peters, E., Garety, P.A., 2006. Cognitive functioning in delusions: a longitudinal analysis Behaviour Research and Therapy 44, 481-514.

Peters, E.R., Joseph, S.A., Garety, P.A., 1999. Measurement of delusional ideation in the normal population: introducing the PDI (Peters et al. Delusions Inventory). Schizophrenia Bulletin 25, 553-576.

Peters, E., Joseph, S., Day, S., Garety, P.A., 2004. Measuring delusional ideation: the 21-item PDI Peters et al. Delusions Inventory. Schizophrenia Bulletin 30 1005-1022.

Peters, E.R., Thornton, P., Siksou, L., Linney, Y., MacCabe, J.H., 2008. Specificity of the jump-to-conclusions bias in deluded patients. The British Journal of Clinical Psychology 47, 239-244.

Pössel, P., 2009. Cognitive Triad Inventory (CTI): psychometric properties and factor structure of the German translation. Journal of Behavior Therapy and Experimental Psychiatry 40, 240-247.

Raine, A., 1991. The SPQ: a scale for the assessment of schizotypal personality based on DSM-III-R criteria. Schizophrenia Bulletin 17, 555-564.

Raine, A., Reynolds, C., Lencz, T., Scerbo, A., Triphon, N., Kim, D., 1994. Cognitiveperceptual, interpersonal, and disorganized features of schizotypal personality. Schizophrenia Bulletin 20, 191-201. 
Sadock, B.J., Sadock, V.A., 2003. Signs and symptoms in psychiatry, In: Kaplan, H.I. Sadock, B.J. (Eds.), Synopsis of Psychiatry: Behavioural Sciences/Clinical Psychiatry, 9th ed. Lippincott Williams Wilkins, Baltimore, p. 283.

Smith, B., Fowler, D., Freeman, D., Bebbington, P., Bashforth, H., Garety, P., Dunn, G., Kuipers, E., 2006. Emotion and psychosis: links between depression, self-esteem, negative schematic beliefs and delusions and hallucinations. Schizophrenia Research 86, 181-188.

So, S.H., Freeman, D., Garety, P.A., 2008. Impact of state anxiety on the jumping to conclusions delusion bias. The Australian and New Zealand Journal of Psychiatry 42 879-886.

Spielberger, C.D., Gorsuch, R.L., Lushene, P.R., Vagg, P.R., Jacobs, A.G., 1983. Manual for the State-Trait Anxiety Inventory Form Y. Consulting Psychologists Press, Inc, Palo Alto.

Van Dael, F., Versmissen, D., Janssen, I., Myin-Germeys, I., van Os, J., Krabbendam, L., 2006. Data gathering: biased in psychosis? Schizophrenia Bulletin 32, 341-351.

van Os, J., Verdoux, H., Maurice-Tison, S., Gay, B., Liraud, F., Salamon, R., Bourgeois, M. 1999. Self-reported psychosis-like symptoms and the continuum of psychosis. Social Psychiatry and Psychiatric Epidemiology 34, 459-463.

Verdoux, H., van Os, J., 2002. Psychotic symptoms in non-clinical populations and the continuum of psychosis. Schizophrenia Research 54, 59-65.

Verdoux, H., Maurice-Tison, S., Gay, B., Van Os, J., Salamon, R., Bourgeois, M.L., 1998. A survey of delusional ideation in primary-care patients. Psychological Medicine 28, 127-134.

Verdoux, H., van Os, J., Maurice-Tison, S., Gay, B., Salamon, R., Bourgeois, M.L., 1999 Increased occurrence of depression in psychosis-prone subjects: a follow-up study in primary care settings. Comprehensive Psychiatry 40, 462-468.

Warman, D.M., 2008. Reasoning and delusion proneness, confidence in decisions. The Journal of Nervous and Mental Disease 196, 1-15.
Warman, D.M., Martin, J.M., 2006. Jumping to conclusions and delusion proneness: the impact of emotionally salient stimuli. The Journal of Nervous and Mental Disease 194, 760-765.

Warman, D.M., Lysaker, P.H., Luedtke, B., Martin, J.M., 2010. Self-esteem and delusion proneness. The Journal of Nervous and Mental Disease 198, 455-457.

Wechsler, D., 1997. WAIS-III: Wechsler Adult Intelligence Scale. Administration and Scoring Manual, 3rd edition. Psychological Corporation/ Harcourt Brace, San Antonio.

Wechsler, D., 2000. Manuel de l'Echelle d'intelligence de Wechsler pour Adultes, 3e édition. ECPA, Paris.

White, L.O., Mansell, W., 2009. Failing to ponder? Delusion-prone individuals rush to conclusions. Clinical Psychology \& Psychotherapy 16, 111-124.

Woodward, T.S., Moritz, S., Arnold, M.M., Cuttler, C., Whitman, J.C., Lindsay, D.S., 2006a. Increased hindsight bias in schizophrenia. Neuropsychology 20, 461-467.

Woodward, T.S., Moritz, S. Cuttler, C. Whitman, J., 2006b. The contribution of a cognitive bias against disconfirmatory evidence (BADE) to delusions in schizophrenia. Journal of Clinical and Experimental Neuropsychology 28, 605-617.

Woodward, T.S., Buchy, L., Moritz, S., Liotti, M., 2007. A bias against disconfirmatory evidence is associated with delusion proneness in a nonclinical sample. Schizophrenia Bulletin 33 (4), 1023-1028.

Young, H.F., Bentall, R.P., 1997. Probabilistic reasoning in deluded, depressed and normal subjects: efforts of task difficulty and meaningful versus non-meaningful material. Psychological Medicine 27, 455-465.

Ziegler, M., Rief, W., Werner, S.M., Mehl, S., Lincoln, T.M., 2008. Hasty decision-making in a variety of tasks: does it contribute to the development of delusions? Psychology and Psychotherapy 81 (Pt 3), 237-245. 